\title{
Expression of the GPDH-4 isozyme of sn-glycerol-3-phosphate dehydrogenase in three Drosophila species
}

\author{
TOMASZ M. WILANOWSKI*†, SIMON H. S. YOONG \\ \& J OHN B. GIBSON† \\ $\dagger$ Molecular Genetics and Evolution Group, Research School of Biological Sciences, The Australian National \\ University, G.P.O. Box 475, Canberra, ACT 2601, Australia, †Division of Biochemistry, University of Tasmania, G.P.O. \\ Box 252-58, Hobart, TAS 7001, Australia and §Abteilung III (Genetik), Max-Planck-Institut für Entwicklungsbiologie, \\ Spemannstr. 35/III, D-72076 Tübingen, Germany
}

\begin{abstract}
A fourth recently discovered isozyme of $s n$-glycerol-3-phosphate dehydrogenase (GPDH-4) in Drosophila melanogaster is shown to be a translational product of the Gpdh transcript which contains exons 1 through 7. This transcript was also found in two other Drosophila species, $D$. busckii and D. virilis. In contrast to D. melanogaster and D. busckii, the Gpdh transcript containing exons $1-6$ is absent in $D$. virilis adults. The reason for this difference between $D$. virilis and the two other species is intriguing but remains elusive. We have ruled out the possibility that a replacement of an amino acid residue in exon 7 played any role in generating this interspecific variation.
\end{abstract}

Keywords: alternative splicing, Drosophila, evolution, GPDH, isozyme.

\section{Introduction}

In Drosophila melanogaster the enzyme sn-glycerol3-phosphate dehydrogenase (GPDH, EC 1.1.1.8) is present in three isozymic forms. GPDH-1 is predominant in the adult flight muscle and involved in energy production for flight, whereas GPDH-3 is localized mainly in the fat body tissue, both in larvae and adult abdomens, and is postulated to serve a dual function regulating the cytoplasmic $\mathrm{NAD}^{+} /$ $\mathrm{NADH}$ ratio and providing $s n$-glycerol-3-phosphate as a precursor for lipid biosynthesis (Bewley \& Miller, 1979). GPDH-2 is found in small amounts in those adult tissues where both GPDH-1 and GPDH3 are expressed. As the functional GPDH molecule is a dimer (Collier et al., 1976) it is possible that GPDH-2 is a heterodimer composed of one GPDH1 subunit and one GPDH-3 subunit (Wright \& Shaw, 1969).

$G p d h$ transcripts are generated by alternative ways of processing the pre-mRNA (Cook et al., 1988). The D. melanogaster Gpdh gene has eight exons, a single promoter and multiple polyadenylation signals (Bewley et al., 1989; von Kalm et al., 1989). There are two alternate $3^{\prime}$ exons - exon 7 and exon 8 .

*Correspondence. E-mail: tomasz@rsbs.anu.edu.au
The transcript containing exons 1 through to 6 codes for GPDH-3 and the transcript containing exons 1-6, skipping exon 7 and including exon 8 encodes GPDH-1 (Bewley et al., 1989; von Kalm et al., 1989). The product of translation of the transcript containing exons 1-7 has not been identified, but it has been suggested that this transcript might be coding for a novel isozyme GPDH-4 (Wilanowski et al., 1995).

The amino acid sequence of GPDH has been obtained from a variety of organisms, and shown to be highly conserved over evolutionary time (Bewley et al., 1989). In fruit fly species this conservation extends to the nucleotide sequence (Kwiatowski et al., 1997) and isozyme patterns (Colgan, 1992). Despite this apparent congruity in the patterns of $G p d h$ variation, few data are available on the extent to which the regulation of the gene's expression is conserved.

For example, the GPDH electrophoretic phenotypes of $D$. virilis and $D$. busckii appear to be very similar to that of D. melanogaster (Fig. 1). However, Tominaga et al. (1992) were able to clone only two kinds of $G p d h$ cDNA from a $D$. virilis adult cDNA library - one contained exons $1-7$ and the second exons $1-6$ and 8 . This result suggests that, unlike in D. melanogaster, the GPDH-3-encoding transcript 
might not be expressed in D. virilis adults. Recently, Wilanowski et al. (1995) have shown that in the $D$. melanogaster $G p d h^{A K y 33}$ allele the insertion of the blood retrotransposon into the noncoding part of exon 7 induces the production of a novel isozyme, GPDH-4, and causes an over-expression of the Gpdh transcript which contains exons 1-7. The comparison between $D$. virilis and $D$. melanogaster poses a number of questions regarding the evolution of Gpdh regulation. We have attempted to answer some of these questions by using P-element mediated transformation in D. melanogaster of a construct containing exons 1-7 and by investigating $G$ pdh expression in D. virilis and D. busckii.

\section{Materials and methods}

Drosophila strains

Wild-type strains of $D$. virilis and $D$. busckii were provided by Allan R. Lohe. The D. melanogaster mutant allele $G p d h^{A K y 33}$ was isolated from a natural population at Cardwell, Queensland, Australia, in

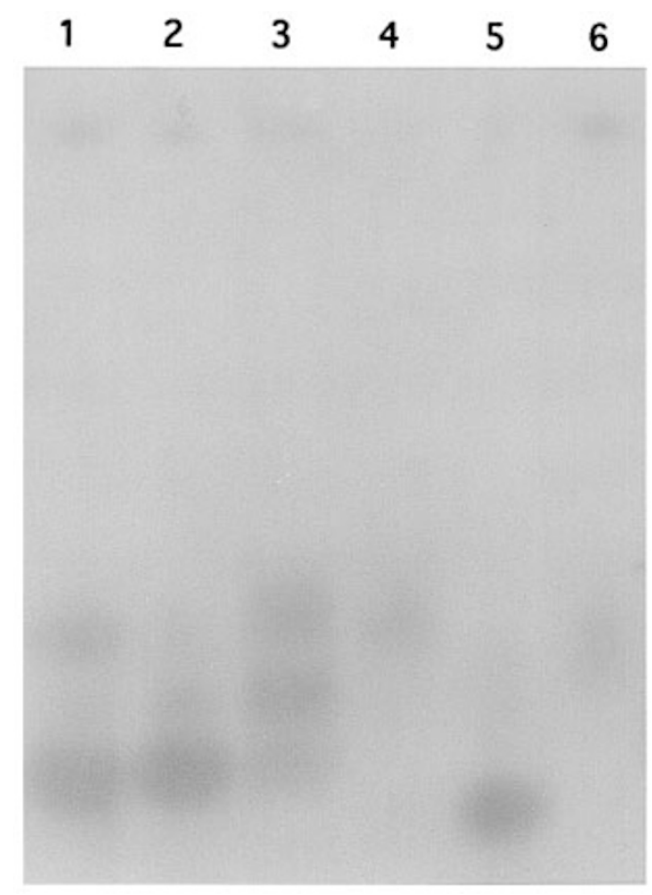

Fig. 1 Cellulose acetate GPDH electrophoretic phenotypes of Drosophila virilis and D. busckii in comparison with $D$. melanogaster. The cathode $(+)$ is at the top of the gel; in $\mathrm{pH} 6.6$ buffer the GPDH protein is negatively charged and migrates towards the anode. Lane 1, D. melanogaster $G p d h^{S}$ (slow) allele whole adult fly. Lanes $2-4, D$. virilis: 2 , whole adult fly; 3 , adult abdomen; 4 , larva. Lanes 5-6, D. busckii: 5, whole adult fly; 6, larva.
1988 (Gibson et al., 1991). A D. melanogaster stock bearing a $G p d h$ null allele, $G p d h^{n J H 231}$, on a balancer chromosome $\mathrm{CyO}$ (Bewley \& Lucchesi, 1977) was obtained from the Bowling Green Stock Centre. $D f$ (2L)Gdh ${ }^{A}$ is a deficiency that spans the Gpdh locus (Lindsley \& Grell, 1968). Flies heterozygous $G p d h^{n J H 231} / D f(2 L) G d h^{A}$ have no detectable GPDH activity and very poor viability.

\section{Gpdh gene constructs and transformation}

Two overlapping genomic clones which cover the Gpdh locus, $p D m 59$ and pDm60, were provided by the late Glenn C. Bewley (Cook et al., 1986). From these clones a construct, $p G p d h(1-7)$, was made to include exons 1-7, the promoter and the $3^{\prime}$ sequences necessary for polyadenylation (Bartoszewski, 1996). The region located downstream of the HindIII site in the noncoding part of exon 6 was replaced by a PCR-amplified fragment covering the entire exon 7. The fragment extends from 91 nucleotides upstream of exon 7 to 48 nucleotides upstream of exon 8. The splice sites between exons 6 and 7 are retained in the $p G p d h(1-7)$ construct, but the entire exon 8 is removed (Fig. 2). The polyadenylation signals in exon 6 are located $3^{\prime}$ to the HindIII site so that they too are removed.

It is known that there is a competition between the reactions of splicing and cleavage-polyadenylation and if the competition from one pre-mRNA processing pathway is eliminated then the other pathway is exclusively followed by default (Peterson et al., 1994). In the $p G p d h(1-7)$ construct effective polyadenylation can occur only in exon 7 as the only polyadenylation signals retained in this construct are those in exon 7. Consequently exon 7 is expected to be spliced to exon 6 as the splice sites in exons 6 and 7 are intact and the competing polyadenylation signals of exon 6 are removed. Thus the only mature Gpdh transcript that can be expressed from this construct is the one which contains exon 7 (Fig. 2). The $p G p d h(1-7)$ construct is not able to produce GPDH-1 or GPDH-3.

P-element mediated transformation, with a wildtype white gene as marker, was used to obtain transformants. The $p G p d h(1-7)$ construct was subcloned into the $p W 8$ vector (Klemenz et al., 1987) and transformed into $D$. melanogaster embryos by microinjection (Bartoszewski \& Gibson, 1994); the recipient strain was homozygous for white eye colour. All the experimental procedures involved in making the construct and in transforming it into D. melanogaster have been described in detail by Bartoszewski (1996). 


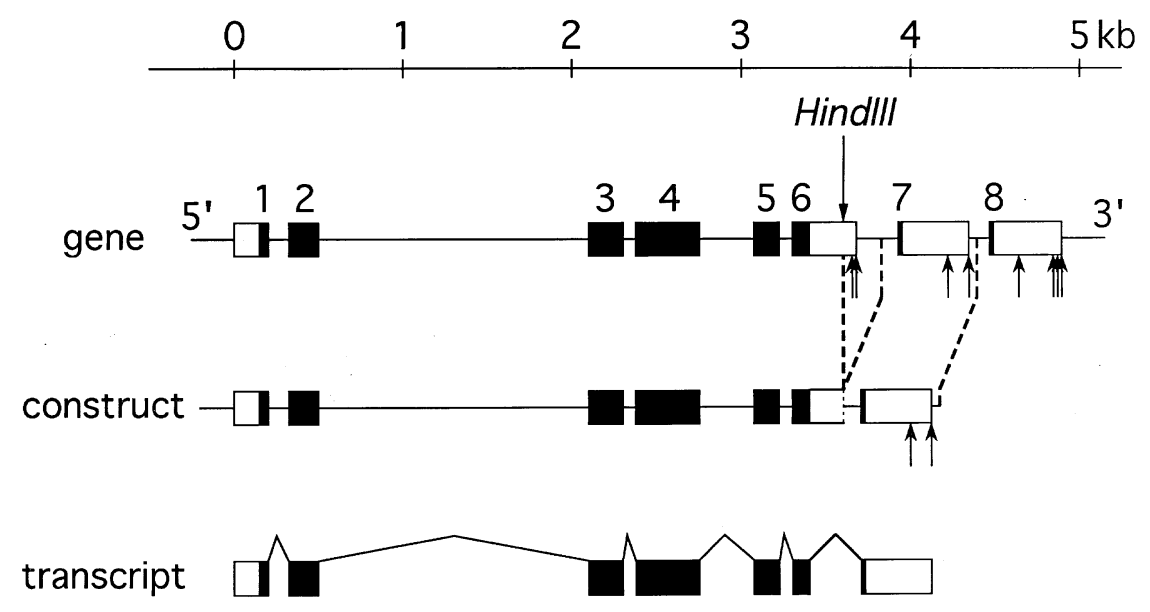

Fig. 2 The structure of the Drosophila melanogaster Gpdh gene (upper diagram) is shown according to Bewley et al. (1989) and von Kalm et al. (1989). Solid boxes indicate the coding regions, and open boxes denote the transcribed but untranslated regions. Arrows below the upper diagram show the locations of putative polyadenylation signals. In the construct $p G p d h(1-7)$ the part of the cloned Gpdh gene located downstream of the HindIII restriction site in exon 6 was replaced with a PCR amplified fragment covering the entire exon 7 (middle). After this modification the only mature transcript (lower) that can be expressed is the one containing exon 7 , because the only polyadenylation signals retained in the $p G p d h(1-7)$ construct are located in exon 7.

\section{GPDH electrophoresis}

GPDH electrophoresis on cellulose acetate membranes with a buffer at $\mathrm{pH} 6.6$ was performed according to Gibson et al. (1986).

Rapid amplification of cDNA ends (RACE) and identification of RACE products

The RACE analysis of the 3' ends of Gpdh transcripts was carried out as previously described (Symonds et al., 1995). A primer specific to exon 5 of the $D$. virilis Gpdh gene, JG119 (5'-CGGGCAAGACAATCGAAGAT-3') was based on the sequence published by Tominaga et al. (1992). For the study of D. busckii we used the JG11 primer (5'-AGAAGCTGCAGGGCCCACCCA-3'), which is specific to exon 5 of the D. melanogaster Gpdh gene. The RACE products obtained were subcloned into pBluescript as before (Wilanowski et al., 1995) and identified by sequencing with the ABI Prism dye terminator cycle sequencing reaction kit (PerkinElmer) according to the manufacturer's instructions.

\section{Results}

The $p G p d h(1-7)$ construct was expressed in the $D$. melanogaster flies made heterozygous $G p d h^{n J H 231} / D f$ (2L) $G d h^{A}$ so that they lack endogenous GPDH activity (Bewley \& Lucchesi, 1977). A number of separately produced transformant lines were obtained but each displayed identical GPDH electrophoretic phenotypes. The phenotype was compared with those of the $G p d h^{F}$ and $G p d h^{A K y 33}$ alleles. As predicted, the transgenic flies express only one GPDH isozyme, which has the same electrophoretic mobility as GPDH-4 in $G p d h^{A K y 33}$ (Fig. 3). This result very strongly supports the hypothesis that this isozyme, named GPDH-4, is a product of translation of a $G p d h$ transcript containing exons 1 through 7 (Wilanowski et al., 1995).

Three RACE product bands were obtained from D. virilis: two both from adults and larvae, with one

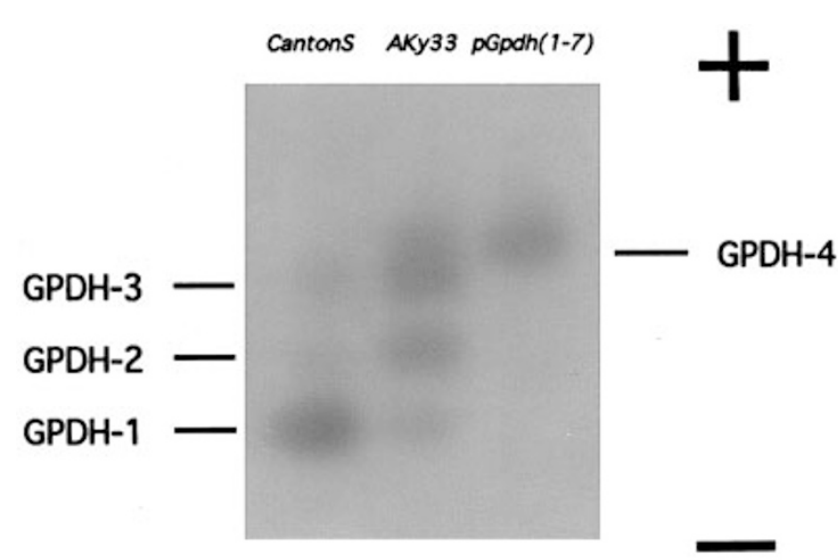

Fig. 3 Electrophoresis on a cellulose acetate membrane of GPDH from adult Drosophila melanogaster transformed with the $p G p d h(1-7)$ construct. Samples from the left: the Canton $S$ stock which carries the $G p d h^{F}$ (fast) allele, the $G p d h^{A K y 33}$ allele, heterozygotes $G p d h^{n J H 231} / D f(2 L) G d h^{A}$ transformed with $p G p d h(1-7)$.

(C) The Genetical Society of Great Britain, Heredity, 81, 390-395. 


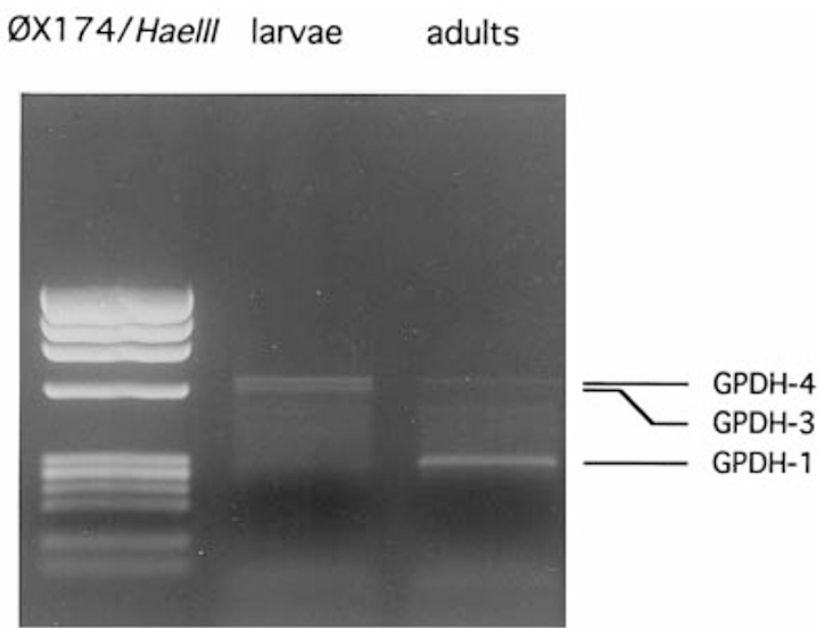

Fig. 4 Two percentage agarose gel showing the results of the RACE experiment on Drosophila virilis. Samples from the left: size standard, RACE products amplified from larvae and adult flies. The intensities of the bands are related not only to the abundance of the corresponding transcripts but to the sizes of the RACE products as well.

band common to both life stages (Fig. 4). All the fragments visible in Fig. 4 have been cloned and sequenced. The band expressed in larvae and adults was found to represent a transcript containing exon 7 , that is, the equivalent of the GPDH-4-encoding transcript in D. melanogaster. These data also show that the transcript containing exons 1-6 - the homologue of the GPDH-3-encoding transcript - is present in $D$. virilis but only in larvae, whereas the equivalent of the $D$. melanogaster transcript coding for GPDH-1 is unique to the adult stage of $D$. virilis.

In $D$. busckii, initial RACE experiments gave several products (Fig. 5). Upon sequencing, only two of them - indicated in the figure - were found to correspond to Gpdh transcripts; other bands resulted from nonspecific amplification. For example, GenBank searching showed that the $\approx 1 \mathrm{~kb}$ RACE fragment represented a transcript encoding acetyl-coenzyme A carboxylase (EC 6.4.1.2). The sensitivity of the RACE method was low probably because of the use of the JG11 primer (Symonds et al., 1995), which was based on the D. melanogaster Gpdh sequence (Bewley et al., 1989; von Kalm et al., 1989) and had two mismatches in comparison with the D. busckii Gpdh sequence (R. Spencer Wells, personal communication).

Nevertheless, the RACE analysis of D. busckii indicated that the homologue of the GPDH1-encoding transcript was unique to the adult stage and the one coding for GPDH-3 was present both in adults and larvae. This experiment failed to detect a transcript containing exon 7 in $D$. busckii. To test further whether this transcript was present in $D$. busckii, we used a more direct approach. A D. melanogaster exon-7-specific primer, JG99 (5'-GACGGCATGATGGACGTATC-3'), was used with JG11 to PCR amplify only the fragment unique to the transcript containing exons 1-7. By this method we detected this transcript both in adults and larvae of $D$. busckii (data not shown). Our findings are summarized in Table 1.

\section{Discussion}

The GPDH electrophoretic phenotypes of $D$. virilis and $D$. busckii appear to resemble that of $D$. melanogaster (Fig. 1). However, the RACE analyses (Figs 4 and 5) showed that although the pattern of $G p d h$ transcription is similar in D. busckii and $D$. melanogaster, it differs from that in $D$. virilis. In $D$. melanogaster, both from adult and larval cDNA samples, three RACE products are obtained that correspond to the transcripts encoding GPDH-1, GPDH-3 and GPDH-4, respectively (Symonds et al.,

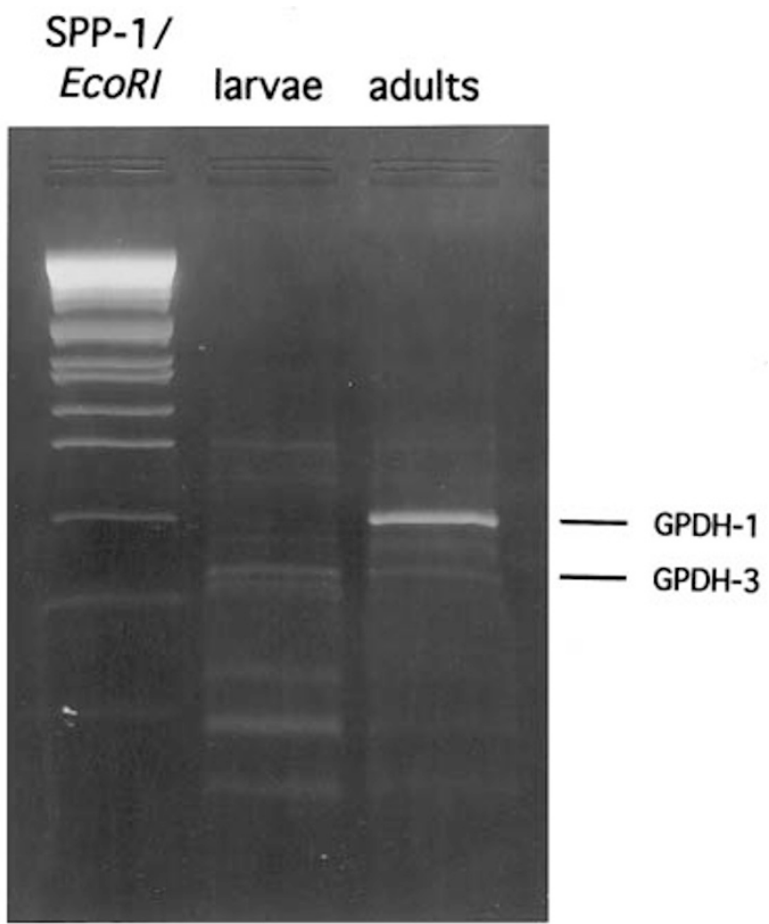

Fig. 5 The results of the RACE experiment on Drosophila busckii. Lanes from the left: size standard, RACE products obtained from larvae and adult flies. The bands confirmed by sequencing to represent $G p d h$ transcripts are indicated; other bands visible in this figure arose from nonspecific amplification. 
Table 1 Comparison of developmental patterns of expression of GPDH isozymes in three Drosophila species

\begin{tabular}{lllll}
\hline Isozyme & RNA structure & D. melanogaster & D. virilis & D. busckii \\
\hline GPDH-1 & Exons 1-6+8 & Adults & Adults & Adults \\
GPDH-2 & None (heterodimer) & Adults & Adults & Adults \\
GPDH-3 & Exons 1-6 & Adults + larvae & Larvae & Adults +larvae \\
GPDH-4 & Exons 1-6 +7 & Only in $G p d h^{A K y 33}$ & Adults +larvae & Adults +larvae \\
\hline
\end{tabular}

1995; Wilanowski et al., 1995). The most significant difference in $D$. virilis is the lack of the GPDH3-encoding transcript in adults. The transcript coding for GPDH-4, however, was found in D. virilis adults (Fig. 4) which suggests that in this species the least anodal isozymic band might be GPDH-4. We base this hypothesis on a comparison between the GPDH electrophoretic phenotype (Fig. 1) and the outcome of the RACE analysis (Fig. 4). In D. virilis the most anodal GPDH staining band and the GPDH-1-encoding transcript are both unique to adults, consequently we argue that this band represents the GPDH-1 isozyme. Thus it is reasonable to assume that the least anodal GPDH staining band in $D$. virilis adults is encoded by the only other $G p d h$ transcript expressed at this stage of development and hence it is the homologue of the GPDH-4 isozyme in D. melanogaster. The middle band in $D$. virilis might then be a heterodimer composed of one GPDH-1 subunit and one GPDH-4 subunit. Similarly, the middle band in $D$. melanogaster and $D$. busckii adults might be a mixture of heterodimers made of one GPDH-1 subunit and one GPDH-3 or GPDH-4 subunit (Wilanowski et al., 1995).

These findings prompt a question about the basis of the variation in Gpdh expression in the Drosophila genus. A comparison of Gpdh sequences obtained from D. melanogaster (Bewley et al., 1989; von Kalm et al., 1989) and D. virilis (Tominaga et al., 1992) revealed that the splice sites in exons 6 and 7 are perfectly conserved but the sequences adjacent to polyadenylation signals in these exons are very different in D. melanogaster and $D$. virilis. This result provides new support to the hypothesis originally formulated by Cook et al. (1988) that the polyadenylation site selection is the regulated event in Gpdh expression.

The change of isozymic patterns could have been triggered by a mutation in exon 7 which is translated only in GPDH-4 (Bewley et al., 1989; von Kalm et al., 1989). There is one amino acid difference between $D$. virilis and $D$. melanogaster in this exon. It is located at residue 353 , which is phenylalanine in D. virilis but serine in D. melanogaster (Tominaga et al., 1992). Drosophila busckii is reputed to be the only other Drosophila species that has the same mutation in exon 7 as D. virilis (Wells, 1996). However, in D. busckii the transcripts coding for GPDH-3 and GPDH-4 are expressed both in adults and larvae, which is different from $D$. virilis (Table 1). It remains possible that some other mutation in the GPDH protein is associated with the shift in the GPDH isozymic pattern. There are seven amino acid replacements in $D$. virilis in comparison with the D. melanogaster $G p d h^{S}$ allele (Tominaga et al., 1992). Unfortunately, as many as three of them are unique to $D$. virilis (Wells 1996) so this supposition is very difficult to test.

The data overall emphasize that seemingly similar phenotypes can be produced by different regulatory patterns in different species. Further studies on the expression of the Gpdh transcripts in various species of Diptera should shed light on this important evolutionary phenomenon.

\section{Acknowledgements}

We thank R. Spencer Wells for providing us with the unpublished nucleotide sequence of the coding region of the Gpdh gene from $D$. busckii, and an anonymous referee for very useful suggestions.

\section{References}

BARtoszewski, s. 1996. Analysis of Regulation of Expression of the sn-Glycerol-3-phosphate Dehydrogenase (Gpdh) Gene of Drosophila melanogaster by P-element Transformation. Ph.D. Thesis, Australian National University.

BARTOSZEWSKI, S. AND GIBSON, J. B. 1994. Injecting un-dechorionated eggs of Drosophila melanogaster under ethanol. Drosoph. Inf. Serv., 75, 205-206.

BEWLEY, G. C. AND LUCCHESI, J. C. 1977. Origin of $\alpha$-glycerophosphate dehydrogenase isozymes in Drosophila melanogaster and their functional relationship in the $\alpha$-glycerophosphate cycle. Biochem. Genet., 15, 235-251.

BEWLEY, G. C. AND MILLER, s. 1979. Origin and differentiation of the soluble $\alpha$-glycerolphosphate dehydrogenase isozymes in Drosophila melanogaster. Isozymes: Curr. Top. Biol. Med. Res., 3, 23-52.

(c) The Genetical Society of Great Britain, Heredity, 81, 390-395. 
BEWLEy, G. C., COOK, J. L., KUSAKABE, S., MUKAI, T., RIGBY, D. L. AND CHAMBERS, G. K. 1989. Sequence, structure and evolution of the gene coding for sn-glycerol-3-phosphate dehydrogenase in Drosophila melanogaster. Nucl. Acids Res., 17, 8553-8567.

COLGAN, D. J. 1992. Glycerol-3-phosphate dehydrogenase isozyme variation in insects. Biol. J. Linn. Soc., 47, 37-47.

COLliER, G. E., SUllivAN, D. T. AND MACINTYRE, R. J. 1976. Purification of $\alpha$-glycerophosphate dehydrogenase from Drosophila melanogaster. Biochim. Biophys. Acta, 429, 316-323.

COOK, J. L., SHAFFER, J. B., BEWLEY, G. C., MACINTYRE, R. J. AND WRIGHT, D. A. 1986. Isolation of a genomic clone for Drosophila sn-glycerol-3-phosphate dehydrogenase using synthetic oligonucleotides. J. Biol. Chem., 261, 11751-11755.

COOK, J. L., BEWLEY, G. C. AND ShAFFer, J. B. 1988. Drosophila sn-glycerol-3-phosphate dehydrogenase isozymes are generated by alternate pathways of RNA processing resulting in different carboxyl-terminal amino acid sequences. J. Biol. Chem., 263, 10858-10864.

GIBSON, J. B., WILKS, A. V., CAO, A. AND FREETH, A. L. 1986. Dominance for $s n$-glycerol-3-phosphate dehydrogenase activity in Drosophila melanogaster: evidence for differential allelic expression mediated via a trans-acting effect. Heredity, 56, 227-235.

GIBSON, J. B., CAO, A., SYMONDS, J. AND REED, D. 1991. Low activity $s n$-glycerol-3-phosphate dehydrogenase variants in natural populations of Drosophila melanogaster. Heredity, 66, 75-82.

KLEMENZ, R., WEBER, U. AND GEHRING, w. J. 1987. The white gene as a marker in a new P-element vector for gene transfer in Drosophila. Nucl. Acids Res., 15, 3947-3959.
KWIATOWSKI, J., KRAWCZYK, M., JAWORSKI, M., SKARECKY, D. AND AYALA, F. J. 1997. Erratic evolution of glycerol3-phosphate dehydrogenase in Drosophila, Chymomyza, and Ceratitis. J. Mol. Evol., 44, 9-22.

LINDSLEY, D. L. AND GRELL, E. H. 1968. Genetic variations of Drosophila melanogaster. Publ. Carnegie Inst. Wash., Number 627.

PETERSON, M. L., BRYMAN, M. B., PEITER, M. AND COWAN, C. 1994. Exon size affects competition between splicing and cleavage-polyadenylation in the immunoglobulin $\mu$ gene. Mol. Cell. Biol., 14, 77-86.

SYMONDS, J. E., GIBSON, J. B., WILKS, A. V. AND WILANOWSKI, T. M. 1995. Molecular analysis of a Drosophila melanogaster sn-glycerol-3-phosphate dehydrogenase allozyme variant that has cold labile activity. Insect Biochem. Mol. Biol., 25, 789-798.

TOMINAGA, H., SHIBA, T. AND NARISE, s. 1992. Structure of Drosophila virilis glycerol-3-phosphate dehydrogenase gene and a comparison with the Drosophila melanogaster gene. Biochim. Biophys. Acta, 1131, 233-238.

VON KALM, L., WEAVER, J., DEMARCO, J., MACINTYRE, R. J. AND SUlLIVAN, D. T. 1989. Structural characterization of the $\alpha$-glycerol-3-phosphate dehydrogenase-encoding gene of Drosophila melanogaster. Proc. Natl. Acad. Sci. U.S.A., 86, 5020-5024.

WELls, R. S. 1996. Nucleotide variation at the Gpdh locus in the genus Drosophila. Genetics, 143, 375-384.

WILANOWSKI, T. M., GIBSON, J. B. AND SYMONDS, J. E. 1995. Retrotransposon insertion induces an isozyme of sn-glycerol-3-phosphate dehydrogenase in Drosophila melanogaster. Proc. Natl. Acad. Sci. U.S.A., 92, 12065-12069.

WRIGHT, D. A. AND SHAw, C. R. 1969. Genetics and ontogeny of $\alpha$-glycerophosphate dehydrogenase isozymes in Drosophila melanogaster. Biochem.. Genet., 3, 343-353. 